\title{
Computation of a Canonical Form for Linear 2D Systems
}

\author{
Mohamed Salah Boudellioua \\ Department of Mathematics and Statistics, Sultan Qaboos University, P.O. Box 36, Al-Khodh, 123 Muscat, Oman
}

Correspondence should be addressed to Mohamed Salah Boudellioua; boudell@squ.edu.om

Received 8 July 2014; Revised 19 November 2014; Accepted 27 November 2014; Published 15 December 2014

Academic Editor: Anh-Huy Phan

Copyright (c) 2014 Mohamed Salah Boudellioua. This is an open access article distributed under the Creative Commons Attribution License, which permits unrestricted use, distribution, and reproduction in any medium, provided the original work is properly cited.

\begin{abstract}
Symbolic computation techniques are used to obtain a canonical form for polynomial matrices arising from discrete 2D linear state-space systems. The canonical form can be regarded as an extension of the companion form often encountered in the theory of 1D linear systems. Using previous results obtained by Boudellioua and Quadrat (2010) on the reduction by equivalence to Smith form, the exact connection between the original polynomial matrix and the reduced canonical form is set out. An example is given to illustrate the computational aspects involved.
\end{abstract}

\section{Introduction}

Canonical forms play an important role in the modern theory of linear systems. In particular, the so-called companion matrix has been used by many authors in the analysis and synthesis of $1 \mathrm{D}$ linear control systems. For instance, Barnett [1] showed that many of the concepts encountered in 1D linear systems theory such as controllability, observability, stability, and pole assignment can be nicely linked via the companion matrix. Boudellioua [2] suggested a matrix form which can be regarded as a $2 \mathrm{D}$ companion form for a class of bivariate polynomials. These polynomials arise in the study of $2 \mathrm{D}$ linear discrete state-space systems describing, for example, 2D image processing systems, as suggested by Roesser [3]. However in that paper, the author did not establish the exact connection between the original matrix and the reduced canonical form. In this paper, using symbolic computation based on the OreModules [4] Maple package the connection between the original polynomial matrix and the canonical form is established.

\section{Polynomial Matrices Arising from Linear 2D Systems}

A $2 \mathrm{D}$ system is a system in which information propagates in two independent directions. These systems arise from applications such as image processing and iterative circuits. Several authors (Attasi [5], Fornasini and Marchesini [6], and Roesser [3]) have proposed different state-space models for 2D discrete linear systems. However, it has been shown that Roesser's model is the most satisfactory and the most general model since the other models can be embedded in it. The model of Roesser is one in which the local state is divided into horizontal and vertical states which are propagated, respectively, horizontally and vertically by first order difference equations. The model has the form:

$$
\begin{aligned}
& x^{h}(i+1, j)=A_{1} x^{h}(i, j)+A_{2} x^{v}(i, j)+B_{1} u(i, j) \\
& x^{v}(i, j+1)=A_{3} x^{h}(i, j)+A_{4} x^{v}(i, j)+B_{2} u(i, j),
\end{aligned}
$$

where $x^{h}(i, j)$ is the horizontal state vector, $x^{v}(i, j)$ is the vertical state vector, $u(i, j)$ is the input vector, and $A_{1}, A_{2}$, $A_{3}, A_{4}, B_{1}$, and $B_{2}$ are real constant matrices of appropriate dimensions. System (1) can be written in the polynomial form:

$$
\left(\begin{array}{cc}
s I_{n}-A_{1} & -A_{2} \\
-A_{3} & z I_{m}-A_{4}
\end{array}\right)\left(\begin{array}{c}
x^{h}(i, j) \\
x^{v}(i, j)
\end{array}\right)=\left(\begin{array}{l}
B_{1} \\
B_{2}
\end{array}\right) u(i, j),
$$

where $s$ represents an advance operator in the horizontal direction and $z$ represents an advance operator in the vertical direction. The polynomial matrix over $\mathbb{R}[s, z]$,

$$
T(s, z)=\left(\begin{array}{cc}
s I_{n}-A_{1} & -A_{2} \\
-A_{3} & z I_{m}-A_{4}
\end{array}\right),
$$

is the characteristic matrix associated with (1). Throughout this paper, unless specified otherwise, $D=K\left[x_{1}, \ldots, x_{n}\right]$ 
denotes the polynomial ring in the indeterminates $x_{1}, \ldots, x_{n}$ with coefficients in an arbitrary but fixed field $K$. First we present a few definitions that will be needed later in the paper.

\section{Definitions}

Definition 1. Let $D=K\left[x_{1}, \ldots, x_{n}\right]$. The general linear group $\mathrm{GL}_{p}(D)$ is defined by

$$
\mathrm{GL}_{p}(D)=\left\{M \in D^{p \times p} \mid \exists N \in D^{p \times p}: M N=N M=I_{p}\right\} .
$$

An element $M \in \mathrm{GL}_{p}(D)$ is called a unimodular matrix. It follows that $M$ is unimodular if and only if the determinant of $M$ is invertible in $D$ that is $|M|$ is a non-zero is a non-zero element of $K$.

One of standard tasks carried out in systems theory is to transform a given system representation into a simpler form before applying any analytical or numerical method. The transformation involved must of course preserve relevant system properties if conclusions about the reduced system are to remain valid about the original one. An equivalence transformation used in the context of multidimensional systems is unimodular equivalence. This transformation can be regarded as an extension of Rosenbrock's equivalence [7] from the univariate to the multivariate setting and is defined by as follows.

Definition 2. Let $T_{1}$ and $T_{2}$ denote two $q \times p$ matrices with elements in $D$; then, $T_{1}$ and $T_{2}$ are said to be unimodular equivalent if there exist two matrices $M \in \mathrm{GL}_{q}(D)$ and $N \in$ $\mathrm{GL}_{p}(D)$ such that

$$
T_{2}=M T_{1} N
$$

\section{Equivalence to Smith Form over$$
D=K\left[x_{1}, \ldots, x_{n}\right]
$$

The Smith form $S$ of a $p \times q$ matrix $P$ with elements in a domain $D$ is usually the result of an equivalence transformation, that is, a transformation of the form

$$
S=M T N \text {, }
$$

where $M$ and $N$ are unimodular matrices with elements in $D$, that is, square with determinant a unit of $D$. The resulting Smith form $S$ is given by

$$
\begin{gathered}
{\left[\begin{array}{ll}
\mathfrak{D} & 0
\end{array}\right] ; \quad(p<q),} \\
\mathfrak{D} ; \quad(p=q), \\
{\left[\begin{array}{c}
\mathfrak{D} \\
0
\end{array}\right] ; \quad(p>q),}
\end{gathered}
$$

where $\mathfrak{D}$ is a $t \times t$ diagonal matrix given by

$$
\mathfrak{D}=\operatorname{diag}\left(\Phi_{1}, \Phi_{2}, \ldots, \Phi_{r}, 0,0, \ldots, 0\right),
$$

$t=\min (p, q)$, and $r=\operatorname{rank}$ of $T$, and the invariant polynomials $\Phi_{i}$ in (8) are given by

$$
\Phi_{i}=\frac{d_{i}}{d_{i-1}}, \quad i=1,2, \ldots, r,
$$

$d_{0}=1$ and $d_{i}$ is the greatest common divisor of the $i$ th order minors of $T$. In order to show that any matrix can be brought by an equivalence transformation to its Smith form, it is usually required that $D$ is a principal ideal domain or a Euclidean domain. The problem of equivalence of a multivariate polynomial matrix to its Smith form was first studied by Frost and Storey [8] who proposed only necessary conditions. Later Frost and Boudellioua [9] presented necessary and sufficient conditions for a class of bivariate polynomial matrices. Lee and Zak [10] also gave some necessary and sufficient conditions in terms of solutions of some polynomial equations. However, these conditions are difficult to test. Lin et al. [11] extended the result in [9] to the multivariate case and Boudellioua and Quadrat [12] generalized it to a larger class of matrices using a module theoretic approach. The establishment of the equivalence to the Smith form is based on the application of the well known Quillen-Suslin Theorem. For the implementation of Quillen-Suslin Theorem on Maple and applications to multidimensional systems theory, the reader is referred to the paper by Fabianska and Quadrat [13].

Theorem 3 (Quillen and Suslin $[14,15]$ ). Let $K$ be a principal ideal domain and $D=K\left[x_{1}, \ldots, x_{n}\right]$ and let $R \in D^{q \times p}$ be a matrix which admits a right-inverse $\widetilde{R} \in D^{p \times q}$; that is, $R \widetilde{R}=I_{q}$. Then, there exists a unimodular matrix $N \in G L_{p}(D)$ such that

$$
R N=\left(\begin{array}{ll}
I_{q} & 0
\end{array}\right) .
$$

Now we state the necessary and sufficient conditions for the reduction of a class of polynomial matrices to the Smith form.

Theorem 4 (see $[9,11,12])$. Let $T \in D^{p \times p}$, with full row rank; then, $T$ is unimodular equivalent to the Smith form:

$$
S=\left(\begin{array}{cc}
I_{p-1} & 0 \\
0 & |T|
\end{array}\right)
$$

where $|T|=\operatorname{det}(T) \in D$ if and only if there exist a vector $U \in D^{p}$ which admits left inverse over $D$ such that the matrix $\left(\begin{array}{ll}T & U\end{array}\right)$ has right inverse over $D$.

\section{Canonical Form for Linear 2D Systems}

Now let $D=\mathbb{R}[s, z]$ and suppose now that there exists a vector $U \in D^{n+m}$ such that the condition in Theorem 4 is satisfied. Then, it follows that the matrix $T$ is equivalent over $D$ to the Smith form:

$$
S=\left(\begin{array}{cc}
I_{n+m-1} & 0 \\
0 & |T|
\end{array}\right)
$$

where

$$
|T|=\left|\begin{array}{cc}
s I_{n}-A_{1} & -A_{2} \\
-A_{3} & z I_{m}-A_{4}
\end{array}\right|
$$

is the $2 \mathrm{D}$ characteristic polynomial of the matrix $A=$ $\left(\begin{array}{ll}A_{1} & A_{2} \\ A_{3} & A_{4}\end{array}\right)$. 
Introduce the canonical form given in [2] for a matrix $T$ in form (3) and let

$$
\begin{aligned}
|T| & =\sum_{j=0}^{m} k_{j}(s) z^{m-j} \\
& =k_{0}(s) z^{m}+k_{1}(s) z^{m-1}+\cdots+k_{m}(s) \\
& =\sum_{i=0}^{n} \sum_{j=0}^{m} P(j, i) s^{n-i} z^{m-j},
\end{aligned}
$$

where $k_{0}(s)$ is monic and has degree equal to $n$ and $k_{j}(s)$ have degrees less or equal to $n,(j=1,2, \ldots, m)$. Consider now the matrix $T_{F} \in D^{(n+m) \times(n+m)}$ in the canonical form:

$$
T_{F}=\left(\begin{array}{cc}
s I_{n}-F_{1} & -\bar{A}_{2} \\
-\bar{A}_{3} & z I_{m}-F_{4}
\end{array}\right),
$$

where $F_{1}$ and $F_{2}$ are in companion form; that is,

$$
\begin{gathered}
F_{1}=\left(\begin{array}{cccc}
0 & 1 & \cdots & 0 \\
0 & 0 & \cdots & 0 \\
\vdots & \vdots & \ddots & \vdots \\
F_{1}(n, n) & F_{1}(n, n-1) & \cdots & F_{1}(n, 1)
\end{array}\right), \\
F_{4}=\left(\begin{array}{cccc}
0 & 1 & \cdots & 0 \\
0 & 0 & \cdots & 0 \\
\vdots & \vdots & \ddots & \vdots \\
F_{4}(m, m) & F_{4}(m, m-1) & \cdots & F_{4}(m, 1)
\end{array}\right),
\end{gathered}
$$

$\bar{A}_{2}=\left(\begin{array}{ll}E_{n} & 0\end{array}\right)$, and $E_{n}$ is the $n$th column of $I_{n}$.

Equating the coefficients of the polynomials $|T|$ and $\left|T_{F}\right|$ determines uniquely the matrix $\bar{A}_{3}=\left[a_{i j}\right]$. Furthermore, using the results given in [2], we get

$$
\begin{gathered}
F_{1}(n, i)=-P(0, n-i+1), \quad i=1,2, \ldots, n, \\
F_{4}(m, j)=-P(m-j+1,0), \quad j=1,2, \ldots, m, \\
\bar{A}_{3}(i, j)=P(i, 0) P(0, n-j+1) \\
-P(i, n-j+1)-\sum_{k=1}^{i-1} P(i-k, 0) \bar{A}_{3}(k, j) .
\end{gathered}
$$

It should be noted here that the unimodular equivalence of a system described by polynomial matrix $T$ in (3), satisfying the condition in Theorem 4, means that such a system can be reduced to an equivalent presentation involving only one single equation in one unknown function. Furthermore, the class of 2D linear systems in (1) amenable to be reduced to the canonical form described above are those which are strongly controllable as studied by Zerz [16, page 75].

Lemma 5. The matrix in the canonical form $T_{F}$ in (15) is unimodular equivalent to the Smith form (12).

Proof. Consider the matrix $T_{F}$ in the canonical form (15), the vector $U=E_{n+m}$, and the highest order minor $m_{1,2, \ldots, n+m}^{2,3, \ldots, n+m+1}$ formed from the rows $1,2, \ldots, n+m$ and columns $2,3, \ldots, n+$ $m+1$ of the matrix $\left(\begin{array}{cc}T_{F} & E_{n+m}\end{array}\right)$; that is,

$$
m_{1,2, \ldots n+m}^{2,3, \ldots, n+m+1}=\left|\begin{array}{cccc}
-1 & 0 & \cdots & 0 \\
* & -1 & \cdots & 0 \\
\vdots & \vdots & \ddots & \vdots \\
* & * & \cdots & -1
\end{array}\right| .
$$

Clearly the matrix $\left(\begin{array}{cc}T_{F} & U\end{array}\right) \equiv\left(\begin{array}{ll}T_{F} & E_{n+m}\end{array}\right)$ has right inverse over $D$ since it has a highest order minor $m_{1,2, \ldots, n+m}^{2,3, \ldots, n+1}$ equal to $(-1)^{n+m}$. Hence by Theorem 4 , the matrix $T_{F}$ is unimodular equivalent to the Smith form (12):

$$
S=\left(\begin{array}{cc}
I_{n+m-1} & 0 \\
0 & \left|T_{F}\right|
\end{array}\right)
$$

The following result based on the Smith form establishes the connection between a polynomial matrix $T$ in form (3) and its equivalent canonical form $T_{F}$ in (15).

Theorem 6. Let $T$ be a polynomial matrix in form (3) satisfying the condition given in Theorem 4; then $T$, is unimodular equivalent over $D$ to the canonical form $T_{F}$ in (15). Furthermore,

$$
T_{F}=M_{2}^{-1} M_{2} T N_{1} N_{2}^{-1},
$$

where the Smith form $S=M_{1} T N_{1}=M_{2} T_{F} N_{2}$.

Proof. By Theorem 4, $T$ is equivalent to the Smith form $S_{1}$, where $S_{1}(n+m, n+m)=|T|$, and by Lemma $5, T_{F}$ is equivalent to the Smith form $S_{2}$, with $S_{2}(n+m, n+m)=\left|T_{F}\right|$. Since $|T|=\left|T_{F}\right|$, it follows that $T$ and $T_{F}$ are equivalent to the same Smith form; that is, $S_{1}=S_{2}$; that is, there exist matrices $M_{1}, N_{1}, M_{2}, N_{2} \in \mathrm{GL}_{n+m}(D)$ such that $S=M_{1} T N_{1}=$ $M_{2} T_{F} N_{2}$. By transitivity of the unimodular equivalence, $T$ and $T_{F}$ are also equivalent with

$$
T_{F}=M_{2}^{-1} M_{2} T N_{1} N_{2}^{-1} .
$$

\section{Illustrative Example}

Let $D=\mathbb{R}[s, z]$ and

$$
T=\left(\begin{array}{cc|cc}
s+1 & -1 & -1 & 1 \\
1 & s & -1 & 1 \\
\hline 2 & -2 & z+1 & -3 \\
0 & -3 & 2 & z-2
\end{array}\right),
$$

where $|T|=\left(s^{2}+s+1\right) z^{2}+\left(-s^{2}+2 s+1\right) z+4 s^{2}-2 s+4$. Using the equations in (17), the matrix in canonical form $T_{F}$ associated with the polynomial $|T|$ is obtained as

$$
\begin{aligned}
T_{F} & \equiv\left(\begin{array}{cc}
s I_{2}-F_{1} & -\bar{A}_{2} \\
-\bar{A}_{3} & z I_{2}-F_{4}
\end{array}\right) \\
& =\left(\begin{array}{cc|cc}
s & -1 & 0 & 0 \\
1 & s+1 & -1 & 0 \\
\hline 2 & 3 & z & -1 \\
2 & -3 & 4 & z-1
\end{array}\right) .
\end{aligned}
$$


First, we reduce the matrix $T$ to the Smith form $S$; that is, compute $M_{1} \in \mathrm{GL}_{4}(D)$ and $N_{1} \in \mathrm{GL}_{4}(D)$ such that $S=$ $M_{1} T N_{1}$ where $S$ is given by (12).

Using the method given by Boudellioua and Quadrat [12], consider vector $U_{1}=\left(\begin{array}{llll}0 & 0 & -1 & -1\end{array}\right)^{T} \in D^{4}$ and $P_{1}=$ $\left(T U_{1}\right) \in D^{4 \times 5}$. Using the package OreModules in Maple, see Chyzak et al. [4], we can check that $P$ admits right inverse over $D$ and we can compute minimal parametrization $Q_{m} \in$ $D^{5}$ of $P$, where $Q_{m}=\left(Q_{1}^{T} Q_{2}^{T}\right)^{T}$ and $P_{1} Q_{m}=0$,

$$
\begin{aligned}
Q_{m} & =\left(\begin{array}{l}
Q_{1} \\
Q_{2}
\end{array}\right) \\
& =\left(\begin{array}{c}
2 s+2 \\
2 s \\
(z+1) s^{2}+(z+4) s+z+3 \\
(z-1) s^{2}+(z+2) s+z+1 \\
\left(z^{2}-z+4\right) s^{2}+\left(z^{2}+2 z-2\right) s+z^{2}+z+4
\end{array}\right) .
\end{aligned}
$$

Computing the Syzygy Module $K_{1} \in D^{3 \times 4}$ of $Q_{1}$, that is, $K_{1} Q_{1}=0$, gives

$$
K_{1}=\left(\begin{array}{cccc}
2 & 1 & z-1 & -z-1 \\
1 & s & -1 & 1 \\
s+1 & -1 & -1 & 1
\end{array}\right)
$$

where the matrix $Q_{3} \in D^{4 \times 3}$ is the right inverse of $K_{1}$; that is,

$Q_{3}$

$$
=\left(\begin{array}{ccc}
0 & 1 & -1 \\
0 & 1 & -1 \\
-\frac{1}{2} & \frac{3}{2}+\left(\frac{1}{2}+\frac{1}{2} z\right) s & \left(-\frac{1}{2}-\frac{1}{2} z\right) s-2-\frac{1}{2} z \\
-\frac{1}{2} & \frac{3}{2}+\left(-\frac{1}{2}+\frac{1}{2} z\right) s & \left(\frac{1}{2}-\frac{1}{2} z\right) s-1-\frac{1}{2} z
\end{array}\right) .
$$

Thus, the matrix $N_{1}=\left(\begin{array}{ll}Q_{3} & Q_{1}\end{array}\right) \in \mathrm{GL}_{4}(D)$ is given by

$$
N_{1}=\left(\begin{array}{cccc}
0 & 1 & -1 & 2+2 s \\
0 & 1 & -1 & 2 s \\
-\frac{1}{2} & \frac{3}{2}+\left(\frac{1}{2}+\frac{1}{2} z\right) s & \left(-\frac{1}{2}-\frac{1}{2} z\right) s-2-\frac{1}{2} z & (z+1) s^{2}+(4+z) s+z+3 \\
-\frac{1}{2} & \frac{3}{2}+\left(-\frac{1}{2}+\frac{1}{2} z\right) s & \left(\frac{1}{2}-\frac{1}{2} z\right) s-1-\frac{1}{2} z & (z-1) s^{2}+(z+2) s+z+1
\end{array}\right)
$$

The matrix $M_{1}=\left(T Q_{3}-U\right)^{-1} \in \mathrm{GL}_{4}(D)$ is given by

$$
M_{1}=\left(\begin{array}{cccc}
0 & 0 & 1 & -1 \\
0 & 1 & 0 & 0 \\
1 & 0 & 0 & 0 \\
\left(2-\frac{1}{2} z+\frac{1}{2} z^{2}\right) s+\frac{1}{2} z^{2}-1+z & \left(-2+\frac{1}{2} z-\frac{1}{2} z^{2}\right) s+3-\frac{3}{2} z & \frac{1}{2} z-\frac{1}{2} z+1
\end{array}\right)
$$

and it can be easily verified that the matrix $M_{1} T N_{1}$ yields the Smith form:

$$
\begin{aligned}
S & =\left(\begin{array}{cc}
I_{3} & 0 \\
0 & Q_{2}
\end{array}\right) \\
& \equiv\left(\begin{array}{cc}
I_{3} & \left(s^{2}+s+1\right) z^{2}+\left(2 s-s^{2}+1\right) z+4 s^{2}-2 s+4
\end{array}\right),
\end{aligned}
$$

where $Q_{2}=|T|$. Similarly, the matrix $T_{F}$ is reduced to the Smith form $S$; that is, compute $M_{2} \in G L_{4}(D)$ and $N_{2} \in$ $G L_{4}(D)$ such that $S=M_{2} T_{F} N_{2}$ where $S$ is given by (12). Now consider vector $U_{2}=\left(\begin{array}{llll}0 & 0 & 0 & -1\end{array}\right)^{T} \in D^{4}$ and $P_{2}=$ $\left(\begin{array}{ll}T_{F} & U_{2}\end{array}\right) \in D^{4 \times 5}$. Using the package OreModules in Maple, we can check that $P_{2}$ admits right inverse over $D$ and we can compute minimal parametrization $\bar{Q}_{m} \in D^{5}$ of $P_{2}$, where $\bar{Q}_{m}=\left(\bar{Q}_{1}^{T} \bar{Q}_{2}^{T}\right)^{T}$ and $P_{2} \bar{Q}_{m}=0$,

$$
\bar{Q}_{m}=\left(\begin{array}{c}
1 \\
s \\
s^{2}+s+1 \\
s^{2} z+(3+z) s+2+z \\
\frac{\left(z^{2}-z+4\right) s^{2}+\left(z^{2}+2 z-2\right) s+z+4+z^{2}}{2}
\end{array}\right) \text {. }
$$

Computing the Syzygy Module $K_{2} \in D^{3 \times 4}$ of $\bar{Q}_{1}$, that is, $K_{2} \bar{Q}_{1}=0$, gives

$$
K_{2}=\left(\begin{array}{cccc}
2 & 3 & z & -1 \\
1 & s+1 & -1 & 0 \\
s & -1 & 0 & 0
\end{array}\right)
$$


where the matrix $\bar{Q}_{3} \in D^{4 \times 3}$ is the right inverse of $K_{1}$; that is,

$$
\bar{Q}_{3}=\left(\begin{array}{ccc}
0 & 0 & 0 \\
0 & 0 & -1 \\
0 & -1 & -s-1 \\
-1 & -z & -3-z-s z
\end{array}\right)
$$

Thus, the matrix $N_{2}=\left(\begin{array}{ll}\bar{Q}_{3} & \bar{Q}_{1}\end{array}\right) \in \mathrm{GL}_{4}(D)$ is given by

$$
N_{2}=\left(\begin{array}{cccc}
0 & 0 & 0 & 1 \\
0 & 0 & -1 & s \\
0 & -1 & -s-1 & s^{2}+s+1 \\
-1 & -z & -3-z-s z & s^{2} z+(3+z) s+2+z
\end{array}\right) .
$$

The matrix $M_{2}=\left(T \bar{Q}_{3}-U_{2}\right)^{-1} \in \mathrm{GL}_{4}(D)$ is given by

$$
\begin{aligned}
& M_{2} \\
& =\left(\begin{array}{cccc}
0 & 0 & 1 & 0 \\
0 & 1 & 0 & 0 \\
1 & 0 & 0 & 0 \\
z^{2}+2 z-2+\left(z^{2}-z+4\right) s & z^{2}-z+4 & -1+z & 1
\end{array}\right)
\end{aligned}
$$

and it can be easily verified that the matrix $M_{2} T_{F} N_{2}$ yields the Smith form

$$
S=\left(\begin{array}{cc}
I_{3} & 0 \\
0 & \bar{Q}_{2}
\end{array}\right)
$$

$$
\equiv\left(\begin{array}{cc}
I_{3} & 0 \\
0 & \left(s^{2}+s+1\right) z^{2}+\left(2 s-s^{2}+1\right) z+4 s^{2}-2 s+4
\end{array}\right),
$$

where $\bar{Q}_{2}=\left|T_{F}\right|=|T|$. It follows that the matrix $T_{F}$ is related to the matrix $T$ by the following transformation:

$$
T_{F}=M T N
$$

where $M=M_{2}^{-1} M_{1} \in \mathrm{GL}_{4}(D)$ and $N=N_{1} N_{2}^{-1} \in \mathrm{GL}_{4}(D)$ are given by

$$
\begin{aligned}
& M=\left(\begin{array}{cccc}
1 & 0 & 0 & 0 \\
0 & 1 & 0 & 0 \\
0 & 0 & 1 & -1 \\
\left(-2-\frac{1}{2} z^{2}+\frac{1}{2} z\right) s-z+1-\frac{1}{2} z^{2} & -1-z^{2}-\frac{1}{2} z+\left(-2-\frac{1}{2} z^{2}+\frac{1}{2} z\right) s & -\frac{1}{2} z+1 & \frac{1}{2} z
\end{array}\right) \\
& N=\left(\begin{array}{cccc}
3+s & 2+s & -1 & 0 \\
s+1 & 2+s & -1 & 0 \\
\left(\frac{1}{2} z+\frac{1}{2}\right) s^{2}+\left(\frac{5}{2}+z\right) s+\frac{7}{2}+z\left(\frac{1}{2} z+\frac{1}{2}\right) s^{2}+\left(\frac{5}{2}+z\right) s+2+\frac{1}{2} z\left(-\frac{1}{2} z-\frac{1}{2}\right) s-\frac{1}{2} z-\frac{3}{2} & \frac{1}{2} \\
\left(\frac{1}{2} z-\frac{1}{2}\right) s^{2}+\left(\frac{1}{2}+z\right) s+\frac{3}{2}+z\left(\frac{1}{2} z-\frac{1}{2}\right) s^{2}+\left(\frac{1}{2}+z\right) s+1+\frac{1}{2} z\left(-\frac{1}{2} z+\frac{1}{2}\right) s-\frac{1}{2} z-\frac{3}{2} & \frac{1}{2}
\end{array}\right) .
\end{aligned}
$$

\section{Conclusions}

In this paper, the Smith form of a bivariate polynomial matrix together with symbolic computation techniques is used effectively to compute the equivalence transformations that reduce a class of $2 \mathrm{D}$ polynomial matrices to a canonical form. The classes of matrices considered are those amenable to be reduced by unimodular equivalence to a single equation in one unknown function. These matrices arise from 2D Roesser systems which are strongly controllable.

\section{Conflict of Interests}

The author declares that there is no conflict of interests regarding the publication of this paper.

\section{Acknowledgments}

The author wishes to express his thanks to Sultan Qaboos University (Oman) for their support in carrying out this research and Dr. Alban Quadrat for his help with the OreModules Maple package.

\section{References}

[1] S. Barnett, "A matrix circle in linear control theory," Bulletin of the Institute of Mathematics and Its Applications, vol. 12, no. 6, pp. 173-176, 1976.

[2] M. S. Boudellioua, "A canonical matrix representation of 2-D linear discrete systems," Journal for Engineering Sciences, vol. 11, no. 2, pp. 81-90, 1999.

[3] R. P. Roesser, "A discrete state-space model for linear image processing," IEEE Transactions on Automatic Control, vol. 20, no. 1, pp. 1-10, 1975. 
[4] F. Chyzak, A. Quadrat, and D. Robertz, "OreModules: a symbolic package for the study of multidimensional linear systems," in Applications of Time-Delay Systems, J. Chiasson and and J.-J. Loiseau, Eds., vol. 352 of Lecture Notes in Control and Information Sciences, pp. 233-264, Springer, 2007, /http://wwwb.math.rwth-aachen.de/OreModules.

[5] S. Attasi, "Systemes lineaires a deux indices," Tech. Rep. 31, IRIA, Paris, France, 1973.

[6] E. Fornasini and G. Marchesini, "State-space realization theory of two-dimensional filters," IEEE Transactions on Automatic Control, vol. 21, no. 4, pp. 484-492, 1976.

[7] H. H. Rosenbrock, State-Space and Multivariable Theory, John Wiley \& Sons, London, UK, 1970.

[8] M. G. Frost and C. Storey, "Equivalence of a matrix over $\mathbb{R}[s$; $z$ ] with its Smith form," International Journal of Control, vol. 28, no. 5, pp. 665-671, 1978.

[9] M. G. Frost and M. S. Boudellioua, "Some further results concerning matrices with elements in a polynomial ring," International Journal of Control, vol. 43, no. 5, pp. 1543-1555, 1986.

[10] E. B. Lee and S. H. Zak, "Smith forms over $\mathbb{R}\left[z_{1}, z_{2}\right]$ ", IEEE Transactions on Automatic Control, vol. 28, no. 1, pp. 115-118, 1983.

[11] Z. Lin, M. S. Boudellioua, and L. Xu, "On the Equivalence and Factorization of Multivariate Polynomial Matrices," in Proceedings of the IEEE International Symposium on Circuits and Systems, pp. 4911-4914, Island of Kos, Greece, May 2006.

[12] M. S. Boudellioua and A. Quadrat, "Serre's reduction of linear functional systems," Mathematics in Computer Science, vol. 4, no. 2, pp. 289-312, 2010.

[13] A. Fabianska and A. Quadrat, "Applications of the QuillenSuslin theorem in multidimensional systems theory," in Grobner Bases in Control Theory and Signal Processing, H. Park and G. Regensburger, Eds., vol. 3 of Radon Series on Computation and Applied Mathematics, pp. 23-106, de Gruyter publisher, 2007.

[14] D. Quillen, "Projective modules over polynomial rings," Inventiones Mathematicae, vol. 36, pp. 167-171, 1976.

[15] A. A. Suslin, "Projective modules over polynomial rings are free," Soviet Mathematics-Doklady, vol. 17, no. 4, pp. 1160-1164, 1976.

[16] E. Zerz, Topics in Multidimensional Linear Systems Theory, Springer, London, UK, 2000. 


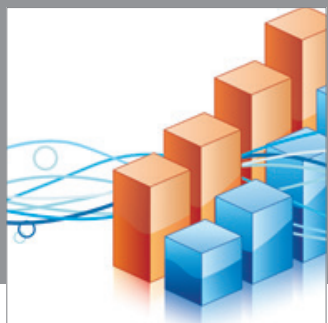

Advances in

Operations Research

mansans

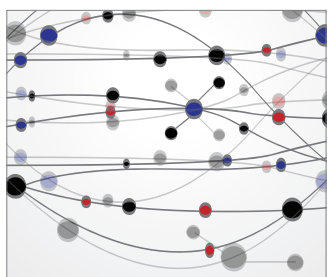

The Scientific World Journal
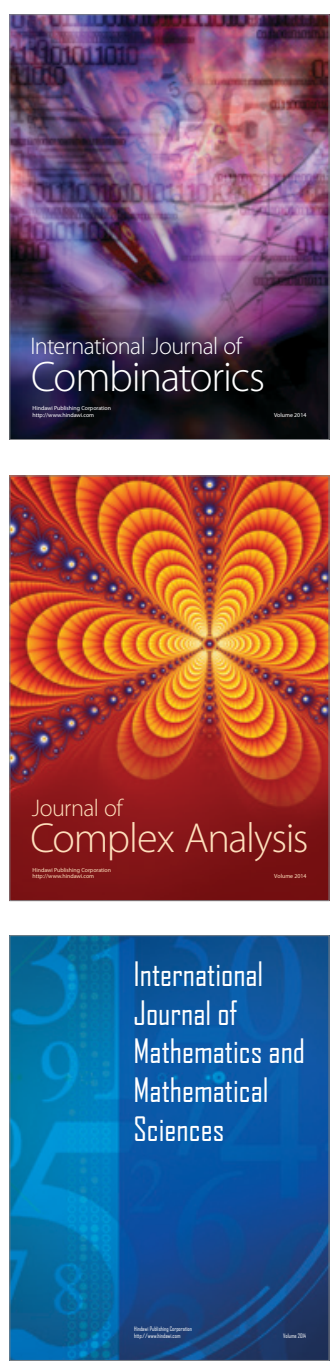
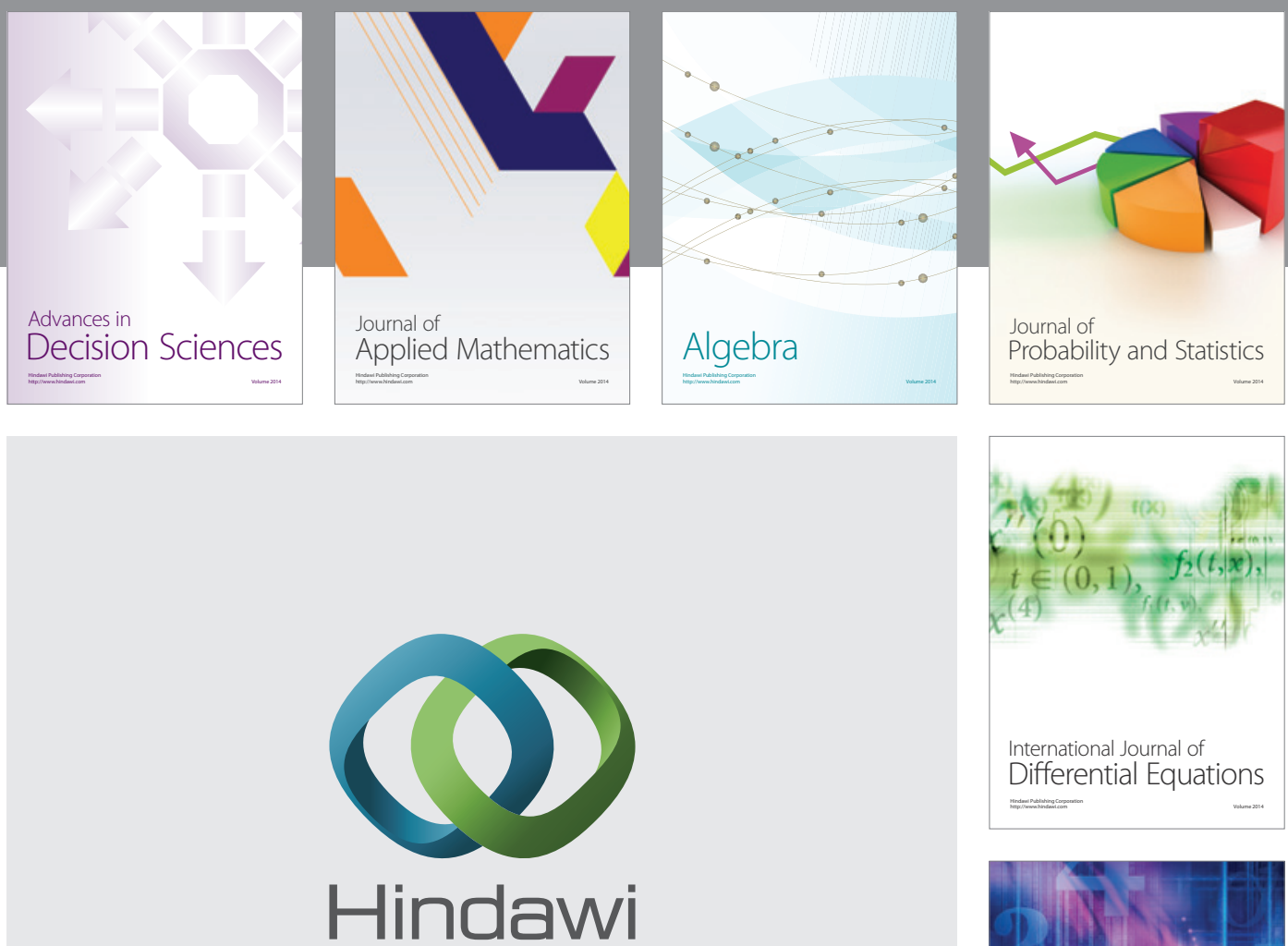

Submit your manuscripts at http://www.hindawi.com
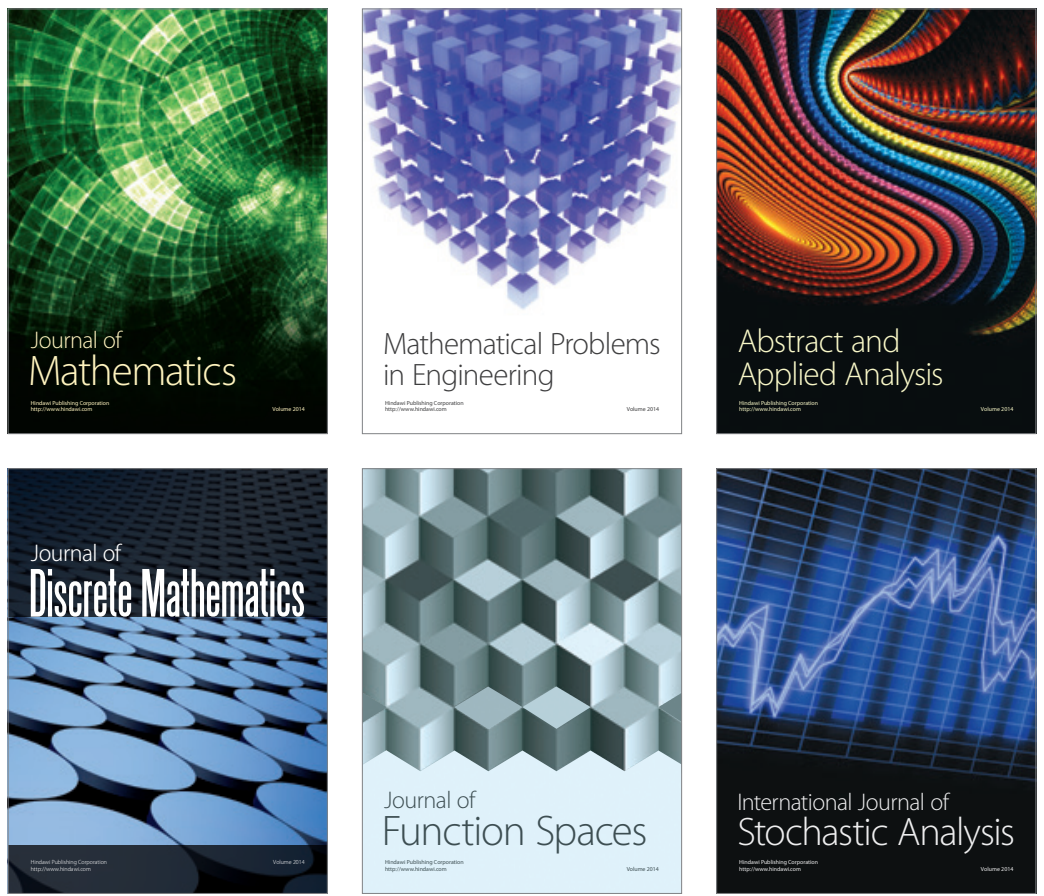

Journal of

Function Spaces

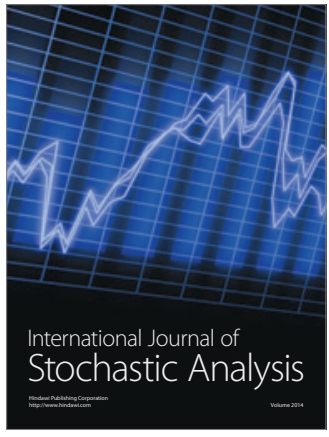

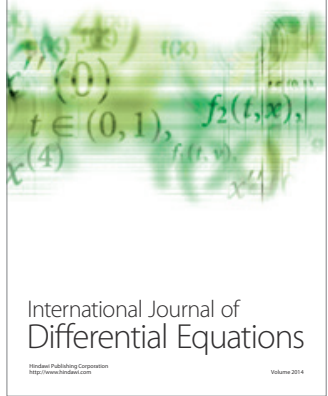
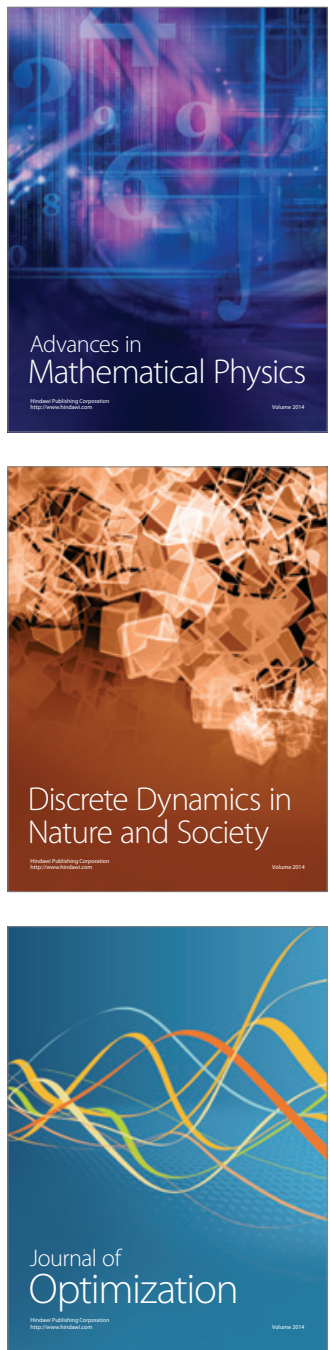